Christian Larsen \& Gudrun P. Kiesmüller

Developing a closed-form cost expression for an $(R, s, n Q)$ policy where the demand process is compound generalized Erlang

Logistics/SCM

Research Group 


\title{
Developing a closed-form cost expression for an $(R, s, n Q)$ policy where the demand process is compound generalized Erlang
}

\author{
Christian Larsen ${ }^{1}$ \\ Department of Business Studies \\ Aarhus School of Business \\ Fuglesangs Allé 4 \\ DK-8210 Aarhus V \\ Denmark \\ Gudrun P. Kiesmüller \\ Faculty of Technology Management \\ Technical University Eindhoven \\ P.O. Box 513 \\ $5600 \mathrm{MB}$ Eindhoven \\ The Netherlands
}

\begin{abstract}
We derive a closed-form cost expression for an $(R, s, n Q)$ inventory control policy where all replenishment orders have a constant lead-time, unfilled demand is backlogged and inter-arrival times of order requests are generalized Erlang distributed. For given values of $Q$ and $R$ we show how to compute the optimal re-order level $s$.

Keywords: Inventory control, compound renewal process, generalized Erlang distribution
\end{abstract}

${ }^{1}$ Corresponding author, e-mail adress chl@asb.dk 


\section{Introduction}

The analysis of an inventory system requires a model for the demand process. Some contributions consider discrete-time models where the total demand in a time period can follow any probability distribution. Others consider only the total demand in a lead-time, often assuming it to be normally distributed. Note, both these approaches, aggregates the demand of the individual order requests. Finally, many contributions are more detailed because here the arrival process of the individual order requests is explicitly modeled. Most often the approach here is to assume the demand process is (compound) Poisson. We refer to [5,8] for a good overview of literature. Concerning the last approach, it is not always reasonable to assume that the time between order requests can be represented by an exponential random variable. For instance, if the stock point is somewhere upstream in the supply chain with a few larger customers, all applying batching policies, it would probably then be more appropriate to model inter-arrival times as a mixture of Erlang distributions. Moreover, it is known (see Theorem 2.9.1 in [6]) that any positive continuous random variable can be arbitrarily closely approximated with a mixture of Erlang distributions, all with the same scale parameter. Therefore it is surprising that this very general demand model is not studied very often.

Inspired by the result of [6] we consider a demand process where the time between order requests is modeled by a random variable $T$ that is a mixture of Erlang distributions, all with the same scale parameter. We assume order requests are modeled by a positive integer-valued random variable $X$. In practice we can not apply the result of [6] in its extreme. We must approximate with a finite number of Erlang distributions. Appendix B in [6] shows two methods to approximate a positive random variable with a mixture of two Erlang distributions, both with the same scale parameter, denoted, by [6], a generalized Erlang distribution. The first method shows how to approximate with a mixture of two Erlang distributions with $k$ and $k+1$ phases, respectively. The second method shows how to approximate with a mixture of an exponential distribution (which is an Erlang distribution with 1 phase) and an Erlang distribution. It is noted by [6] that the first of these methods probably has most practical relevance due to that it will generate a unimodal distribution, which resembles a gamma distribution. Therefore we have decided in the paper to feature the first of these two methods. Thus we assume that the random variable $T$ has the following distribution

$$
T=\left\{\begin{array}{lll}
T_{1} & \text { prob } & p \\
T_{2} & \text { prob } & 1-p
\end{array}\right.
$$

where $T_{1}$ is Erlang distributed with mean $k / \lambda, T_{2}$ is Erlang distributed with mean $(k+1) / \lambda$, $k$ is a positive integer and $\lambda$ is a positive real number.

We analyze an $(R, s, n Q)$ inventory control policy where all replenishment orders have a constant lead-time $L$ and unfilled demand is back-logged. It is a periodic review model, 
where the time between reviews is specified by a given constant $R$. We find it more realistic to consider a periodic review model rather than a continuous one, because in reality there will always be a lower bound on how often it is possible to replenish. The control policy operates as follows. At each review instance, if the inventory position is at or below $s$ then a replenishment order is made with size of an integer multiple of $Q$, bringing the inventory position to be in the interval from $s+1$ to $s+Q$. We assume $Q$ is some predetermined "practical" batch-size, like a pallet or a box, but we have to find $s$. Since $Q$ is given we do not include a fixed replenishment cost into our model, because the expected number of replenishments per time unit is fixed. We will, however, assume that there are given parameters of inventory and penalty costs. We derive a closed-form cost expression of this $(R, s, n Q)$ policy where one subsequently can find the optimal reorder level $s$. We believe that many in the research community shy away from making inventory analyses assuming non-Poisson processes because they think it is either too difficult or impossible to derive closed form cost expressions. Our conclusion is that it is possible and not that difficult though it requires some lengthy mathematical derivations.

In Section 2 we analyze the compound renewal process. In Section 3 we develop our cost expression of the $(R, s, n Q)$ policy and present our algorithm to compute the optimal $s$. In Section 4 we illustrate our derivations by a numerical example. Finally, Section 5 contains some concluding remarks.

\section{Analysis of the renewal process}

One can find some, but not many, expositions on compound renewal processes and inventory theory, cf. [1,4]. Our exposition follows the analysis in [3]. Not all the lengthy mathematical derivations are stated in the paper, but they can be found in the Appendix. Define:

$T(m)$ The random variable which is the sum of $m$ inter-arrival times. Per definition $T(1)$ $\equiv T$ and $T(0) \equiv 0$.

$\Omega_{m}(t)$ The distribution function of $T(m)$. It is the probability that at least $m$ customers have arrived in a time interval of length $t$ that began immediately after a demand instance.

$p_{m}(t)$ The probability that exactly $m$ customers have arrived a time interval of length $t$ that began immediately after a demand instance.

$\tilde{T} \quad$ The random variable which is the residual time from a randomly chosen time point until the next demand instance.

$\tilde{\omega}(t) \quad$ The density function of $\tilde{T}$. 
$\tilde{\Omega}_{m}(t)$ The distribution function of $\tilde{T}+T(m-1)$. It is the probability that at least $m$ customers have arrived in a time interval of length $t$ where the start time is randomly chosen.

$\tilde{p}_{m}(t)$ The probability that exactly $m$ customers have arrived in a time interval of length $t$ where the start time is randomly chosen.

In order to evaluate $\Omega_{m}(t)$ we need to look at all the possible ways in which $m$ interarrival times can be generated. With probability $b(m, i)=\frac{m !}{i !(m-i) !} p^{i}(1-p)^{m-i}$ when generating $m$ inter-arrival times, we generate $i$ inter-arrival times from distribution $T_{1}$ and $m-i$ inter-arrival times from distribution $T_{2}$. In all, the number of phase completions must in this case be at least $i k+(m-i)(k+1)=(k+1) m-i$, which has probability $e^{-\lambda t} \sum_{j=(k+1) m-i}^{\infty} \frac{(\lambda t)^{j}}{j !}$, as all phase completions have durations that are exponentially distributed with mean $1 / \lambda$.

By collecting all these probabilities we get

$\Omega_{m}(t)=e^{-\lambda t} \sum_{i=0}^{m} b(m, i) \sum_{j=(k+1) m-i}^{\infty} \frac{(\lambda t)^{j}}{j !} \quad m=1,2, \ldots$

where per definition $\Omega_{0}(t)=1$.

It follows from [4] that

$$
\tilde{\omega}(t)=\frac{\lambda}{k+1-p} e^{-\lambda t}\left[\sum_{j=0}^{k-1} \frac{(\lambda t)^{j}}{j !}+(1-p) \frac{(\lambda t)^{k}}{k !}\right]
$$

From [4] we also have

$$
\tilde{\Omega}_{m}(t)=\int_{0}^{t} \Omega_{m-1}(t-x) \tilde{\omega}(x) d x
$$

which can be rewritten to:

$$
\tilde{\Omega}_{m}(t)=e^{-\lambda t} \sum_{r=k(m-1)+1}^{(k+1) m-1} \tilde{\Gamma}_{m}(r) \frac{(\lambda t)^{r}}{r !}+e^{-\lambda t} \sum_{r=(k+1) m}^{\infty} \frac{(\lambda t)^{r}}{r !}
$$


where the coefficients $\tilde{\Gamma}_{m}(r)$ are given as follows.

When $k(m-1)+1 \leq r \leq k m$

$\left.\tilde{\Gamma}_{m}(r)=\frac{1}{k+1-p} \sum_{i=\max \{(k+1)(m-1)-r+1,0\}}^{m-1} b(m-1, i)(r-(k+1)(m-1)+i)\right)$

When $k m+1 \leq r \leq(k+1) m-1$

$$
\begin{aligned}
\tilde{\Gamma}_{m}(r)= & \left.\frac{1}{k+1-p} \sum_{i=\max \{(k+1)(m-1)-r+1,0\}}^{n-1-r+k m} b(m-1, i)(r-(k+1)(m-1)+i)\right) \\
& +\sum_{i=(k+1) m-r}^{m-1} b(m-1, i)
\end{aligned}
$$

Here we use the convention that $b(0,0)=1$.

Because $\tilde{p}_{m}(t)=\tilde{\Omega}_{m}(t)-\tilde{\Omega}_{m+1}(t)$ (and thus $\left.\tilde{p}_{0}(t)=1-\tilde{\Omega}_{1}(t)\right)$, it can be written in condensed form as

$$
\tilde{p}_{m}(t)=\left\{\begin{array}{cc}
e^{-\lambda t} \sum_{r=0}^{k} \tilde{\gamma}_{0}(r) \frac{(\lambda t)^{r}}{r !} & m=0 \\
e^{-\lambda t \sum_{r=k(m-1)+1}^{(k+1)(m+1)-1} \tilde{\gamma}_{m}(r) \frac{(\lambda t)^{r}}{r !}} & m=1,2 \ldots
\end{array}\right.
$$

where the coefficients $\tilde{\gamma}_{m}(r)$ are given as follows.

$$
\begin{aligned}
& \tilde{\gamma}_{0}(r)=\left\{\begin{array}{cc}
1 & r=0 \\
1-\tilde{\Gamma}_{0}(r) & 1 \leq r \leq k
\end{array}\right. \\
& \tilde{\gamma}_{1}(r)=\left\{\begin{array}{cc}
\tilde{\Gamma}_{1}(r) & 1 \leq r \leq k \\
1-\tilde{\Gamma}_{2}(r) & k+1 \leq r \leq 2 k+1
\end{array}\right.
\end{aligned}
$$

and for $m \geq 2$ 
$\tilde{\gamma}_{m}(r)=\left\{\begin{array}{cc}\tilde{\Gamma}_{m}(r) & k(m-1)+1 \leq r \leq k m \\ \tilde{\Gamma}_{m}(r)-\tilde{\Gamma}_{m+1}(r) & k m+1 \leq r \leq(k+1) m-1 \\ 1-\tilde{\Gamma}_{m+1}(r) & (k+1) m \leq r \leq(k+1)(m+1)-1\end{array}\right.$

In finishing this section, we also define the random variable:

$\tilde{D}_{(t)} \quad$ the total demand in a time interval of length $t$ with a randomly chosen start time.

It has probability distribution:

$P\left(\tilde{D}_{(t)}=x\right)=\left\{\begin{array}{cc}\tilde{p}_{0}(t) & x=0 \\ \sum_{m=1}^{x} \tilde{p}_{m}(t) P(X(m)=x) & x=1,2, . .\end{array}\right.$

where $X(m)$ is the random variable denoting the total demand of $m$ customers, where per definition $X(1) \equiv X$.

\section{The $(R, s, n Q)$ inventory control policy}

Note that we let the length of the review period be $R$ and not scaled to 1 as in many expositions. We find it most natural to derive the cost expression as generally as possible. In a later contribution we also, as in [7], intend to let this $(R, s, n Q)$ policy be a subpart of a multi-item inventory control policy where the length of the review period is a policy variable. We assume the cost parameters:

$h$ : inventory cost rate

$v$ : penalty cost rate

$\pi$ : fixed cost per item backlogged.

Let $x$ be the inventory position at a review instance just after a replenishment decision has been made (thus $x$ belongs to the interval from $s+1$ to $s+Q$ ). Denote by $G(x)$ the total costs incurred during the review period when starting with an inventory position of $x$. Then following the derivation of the cost function of Model 3 in [3] we get

If $x \leq 0$ 


$$
G(x)=v\left[-x R+\frac{\lambda E[X]}{k+1-p} R\left(L+\frac{R}{2}\right)\right]+\pi \frac{\lambda E[X]}{k+1-p} R
$$

If $x>0$

$$
\begin{aligned}
G(x)= & v\left[-x R+\frac{\lambda E[X]}{k+1-p} R\left(L+\frac{R}{2}\right)\right]+\pi \frac{\lambda E[X]}{k+1-p} R \\
& +(v+h) \sum_{y=0}^{x-1}(x-y) \int_{0}^{R} P\left(\tilde{D}_{(L+\tau)}=y\right) d \tau \\
& \pi \sum_{y=0}^{x-1}(x-y)\left[P\left(\tilde{D}_{(L+R)}=y\right)-P\left(\tilde{D}_{(L)}=y\right)\right]
\end{aligned}
$$

These are the same cost expression as that in [3] (Equation 14). Due to our more numerical approach we have specifically chosen to write everything as finite sums.

In order to make the cost expression computable we still need to evaluate the integral

$$
\int_{0}^{R} P\left(\tilde{D}_{(L+\tau)}=y\right) d \tau
$$

For a Poisson process with rate $\lambda$, let the random variable $N_{A}$ denote the number of arrivals in a time interval of length $A$. It means that $N_{A}$ is Poisson distributed with mean $\lambda A$.

By repeated partial integration it holds that

$$
\int_{0}^{R} e^{-\lambda(L+\tau)} \frac{(\lambda(L+\tau))^{r}}{r !} d \tau=\frac{P\left(N_{L} \leq r\right)-P\left(N_{L+R} \leq r\right)}{\lambda}
$$

Consequently, if $y>0$

$$
\int_{0}^{R} P\left(\tilde{D}_{(L+\tau)}=y\right) d \tau
$$




$$
=\frac{1}{\lambda} \sum_{m=1}^{y} P(X(m)=y) \sum_{r=k(m-1)+1}^{(k+1)(m+1)-1} \tilde{\gamma}_{m}(r)\left[P\left(N_{L} \leq r\right)-P\left(N_{L+R} \leq r\right)\right]
$$

and if $y=0$

$$
\begin{aligned}
\int_{0}^{R} P\left(\tilde{D}_{(L+\tau)}\right. & =y) d \tau \\
& =\frac{1}{\lambda} \sum_{r=0}^{k} \tilde{\gamma}_{0}(r)\left[P\left(N_{L} \leq r\right)-P\left(N_{L+R} \leq r\right)\right]
\end{aligned}
$$

When combining (12a-b) with (8) and (9), we get a closed-form expression of the cost function. The inventory position, immediately after a replenishment decision, is uniformly distributed on $s+1, \ldots, s+Q$. For a mathematical proof see Exercise 6.5 in [8]. Therefore the relevant cost expression to be optimized (with respect to $s$ ) is:

$$
\frac{\sum_{x=s+1}^{s+Q} G(x)}{Q}
$$

This is accomplished by the following algorithm, stated here in pseudo programming language. It is similar to the algorithm in [2].

\section{Algorithm}

$$
\begin{aligned}
& \mathrm{s}:=\operatorname{argmin}\{\mathrm{G}(\mathrm{x}): \mathrm{x} \geq 0\} \\
& \mathrm{s}:=\mathrm{s}-1 \\
& \mathrm{q}:=1 \\
& \text { While } \mathrm{q}<\mathrm{Q} \text { do } \\
& \qquad \text { If } \mathrm{G}(\mathrm{s})<\mathrm{G}(\mathrm{s}+\mathrm{q}+1) \text { then } \\
& \qquad \mathrm{s}:=\mathrm{s}-1
\end{aligned}
$$

End while

End If

$$
\mathrm{q}:=\mathrm{q}+1
$$

\section{End Algorithm}

Each time the while-loop is entered we have found the point's $s+1, \ldots, s+q$, where the function $G(x)$ attains its least values. The justification for this observation follows from [3] who under very general conditions show that $G(x)$ is quasi-convex. Because $q<Q$ we must enlarge this range. If $G(s)<G(s+q+1)$ then we should enlarge this range in a downward direction, and accordingly decrement $s$ by 1 . Otherwise $s$ remains the same. In any case $q$ is incremented by 1 . In this manner we continue until $q=Q$. 


\section{A numerical example}

We have developed a computer code, programmed in Visual Basic for Excel, which computes (9a-b) and finds the optimal value of $s$. In order to demonstrate this we present a numerical example. We have cost parameters $h=2, v=4, \pi=30$. The lead-time $L=$ 0.5 , and the length of review period $R=1$. The characteristics of the demand process is $k$ $=4, p=0.2, \lambda=8$ while the random variable $X$ can attain values $1,2,3$ and 4 with equal probability. Table 1 lists our results for values of $Q$ ranging from 1 to 6 . We have also simulated the inventory system using the computed control policy, because in [3] it is acknowledged that Model 3 is an approximation. Here we believe, however, that [3] is very cautious, which also seems to be tacitly assumed in [3], since otherwise the following rigorous analysis of the cost function in [3] seems odd. We believe that for all practical purposes a review instance must be considered as a randomly chosen time point with respect to the renewal process. Therefore the cost expression of Model 3 in [3] should be almost exact.

\section{$<$ Table 1 about here $>$}

As expected when comparing the simulation results to the derived cost expressions, we see a nice fit in Table 1.

\section{Concluding remarks}

In this paper we have demonstrated how to develop a closed-form cost-expression for an $(R, s, n Q)$ inventory system where the demand process is compound generalized Erlang. Furthermore we also demonstrate how to develop a computational tool to find the optimal reorder level. It is, of course, also possible to make a similar analysis for the case of continuous review, taking a point of departure in Model 2 in [3]. In our paper we have assumed that the order size distribution $X$ is positive and integer valued. We could also have developed our analysis assuming $P(X=0)>0$ as well as assuming $X$ to be a continuous random variable. It is also clear that it is possible to further generalize the model by letting $T$ to be mixtures of more than 2 Erlang distributions. The derived mathematical expressions will then involve multinomial expressions instead of binomial expressions but they remain computable.

\section{Acknowledgement}

This research was made while the first author spent his sabbatical leave at Technical University Eindhoven, The Netherlands, partly supported by a grant from Otto Mønsteds Fond. 


\section{References}

[1] M. Beckmann, An inventory model for arbitrary interval and quantity distributions of demand, Management Science, 8 (1961) 35-57.

[2] A. Federgruen, Y. Zheng, An efficient algorithm to compute an optimal $(r, Q)$ policy in continuous review inventory systems. Operations Research, 40(1992) 808-813.

[3] K. Rosling, Inventory cost rate functions with nonlinear shortage costs. Operations Research, 50 (2002) 1007-1017.

[4] I. Sahin, Regenerative inventory systems: operating characteristics and optimization, Springer, 1990.

[5] E.A. Silver, D.F. Pyke, R. Peterson, Inventory management and production planning and scheduling, Wiley, 1998.

[6] H. Tijms, Stochastic models: an algorithmic approach, Wiley, 1994.

[7] S. Viswanathan, Periodic review $(s, S)$ policies for joint replenishment inventory problems. Management Science, 43 (1997) 1447-1454.

[8] P.H. Zipkin, Foundations of inventory management, McGraw-Hill, 2000. 


\section{Tables}

\begin{tabular}{|c|c|c|c|}
\hline$Q$ & Optimal $s$ & Minimum costs (13) & Simulated costs \\
\hline 1 & 10 & 15.8385 & $15.8469(0.0308)$ \\
\hline 2 & 9 & 15.9479 & $15.9561(0.0408)$ \\
\hline 3 & 9 & 16.1961 & $16.2038(0.0349)$ \\
\hline 4 & 8 & 16.6359 & $16.6326(0.0378)$ \\
\hline 5 & 8 & 16.9368 & $16.9301(0.0317)$ \\
\hline 6 & 8 & 17.4249 & $17.4288(0.0356)$ \\
\hline
\end{tabular}

Table 1 Optimal solutions for the data set when $Q$ ranges from 1 to 6 . The simulation was conducted in Arena, Version 9.0. Each simulation lasted 120000 time units and the initial inventory position and net-inventory were both set equal to $s+Q$. The simulation experiment was replicated 10 times. In parenthesis is stated the $95 \%$ confidence interval. 


\section{Documentation for the derived formulas}

\section{Appendix A}

\section{Derivation of expressions (4b), (5a-c)}

First consider the case $m>1$. Expression (4a) can be written as

$$
\begin{gathered}
\tilde{\Omega}_{m}(t)=\int_{0}^{t}\left[e^{-\lambda(t-x)} \sum_{i=0}^{m-1} b(m-1, i) \sum_{j=(k+1)(m-1)-i}^{\infty} \frac{(\lambda(t-x))^{j}}{j !}\right] \\
{\left[\frac{\lambda}{k+1-p} e^{-\lambda x}\left[\sum_{q=0}^{k-1} \frac{(\lambda x)^{q}}{q !}+(1-p) \frac{(\lambda x)^{k}}{k !}\right]\right] d x} \\
=\frac{\lambda e^{-\lambda t}}{k+1-p}\left[\sum_{i=0}^{m-1} b(m-1, i) \sum_{j=(k+1)(m-1)-i}^{\infty} \sum_{q=0}^{k-1} \int_{0}^{t} \frac{(\lambda(t-x))^{j}}{j !} \frac{(\lambda x)^{q}}{q !} d x\right. \\
\left.+(1-p) \sum_{i=0}^{m-1} b(m-1, i) \sum_{j=(k+1)(m-1)-i}^{\infty} \frac{(\lambda(t-x))^{j}}{j !} \frac{(\lambda x)^{q}}{q !} d x\right]
\end{gathered}
$$

We claim

$$
\int_{0}^{t} \frac{(\lambda(t-x))^{j}}{j !} \frac{(\lambda x)^{q}}{q !} d x=\frac{1}{\lambda} \frac{(\lambda t)^{j+q+1}}{(j+q+1) !}
$$

for all nonnegative integer's $j$ and $q$

It is verified by considering the following three cases.

The case $j=0$ and $q \geq 0$

$$
\int_{0}^{t} \frac{(\lambda x)^{q}}{q !} d x=\frac{1}{\lambda}\left[\frac{(\lambda x)^{q+1}}{(q+1) !}\right]_{0}^{t}=\frac{1}{\lambda} \frac{(\lambda t)^{q+1}}{(q+1) !}
$$

The case $j>0$ and $q=0$ 
$\int_{0}^{t} \frac{(\lambda(t-x))^{j}}{j !} d x=\frac{-1}{\lambda}\left[\frac{(\lambda(t-x))^{j+1}}{(j+1) !}\right]_{0}^{t}=\frac{1}{\lambda} \frac{(\lambda t)^{j+1}}{(j+1) !}$

The case $j>0$ and $q>0$

$\int_{0}^{t} \frac{(\lambda(t-x))^{j}}{j !} \frac{(\lambda x)^{q}}{q !} d x=\left[\frac{-1}{\lambda} \frac{(\lambda(t-x))^{j+1}}{(j+1) !} \frac{(\lambda x)^{q}}{q !}\right]_{0}^{t}+\int_{0}^{t} \frac{(\lambda(t-x))^{j+1}}{(j+1) !} \frac{(\lambda x)^{q-1}}{(q-1) !} d x$

$=\int_{0}^{t} \frac{(\lambda(t-x))^{j+1}}{(j+1) !} \frac{(\lambda x)^{q-1}}{(q-1) !} d x=\ldots . .=\int_{0}^{t} \frac{(\lambda(t-x))^{j+q}}{(j+q) !} d x$

$=\left[\frac{-1}{\lambda} \frac{(\lambda(t-x))^{j+q+1}}{(j+q+1) !}\right]_{0}^{t}=\frac{1}{\lambda} \frac{(\lambda t)^{j+q+1}}{(j+q+1) !}$

Therefore (A1) can be rewritten to

$$
\begin{aligned}
& \tilde{\Omega}_{m}(t)=\frac{e^{-\lambda t}}{k+1-p} {\left[\sum_{i=0}^{m-1} b(m-1, i) \sum_{j=(k+1)(m-1)-i}^{\infty} \sum_{q=1}^{k} \frac{(\lambda t)^{j+q}}{(j+q) !}\right.} \\
&\left.+(1-p) \sum_{i=0}^{m-1} b(m-1, i) \sum_{j=(k+1)(m-1)-i}^{\infty} \frac{(\lambda t)^{j+k+1}}{(j+k+1) !}\right]
\end{aligned}
$$

By introducing a new index $r=j+q$ and collecting all terms with $j+q=r$ it holds for any non-negative integer $u$ that

$$
\sum_{j=u}^{\infty} \sum_{q=1}^{k} \frac{(\lambda t)^{j+q}}{(j+q) !}=\sum_{r=u+1}^{u+k}(r-u) \frac{(\lambda t)^{r}}{r !}+k \sum_{r=u+k+1}^{\infty} \frac{(\lambda t)^{r}}{r !}
$$

which means

$$
\sum_{j=(k+1)(m-1)-i}^{\infty} \sum_{q=1}^{k} \frac{(\lambda t)^{j+q}}{(j+q) !}=\sum_{r=(k+1)(m-1)-i+1}^{(k+1)(m-1)-i+k}(r-(k+1)(m-1)+i) \frac{(\lambda t)^{r}}{r !}+k \sum_{r=(k+1) m-i}^{\infty} \frac{(\lambda t)^{r}}{r !}
$$


Therefore (A3) can be rewritten to

$$
\begin{gathered}
\tilde{\Omega}_{m}(t)=\frac{e^{-\lambda t}}{k+1-} \sum_{i=0}^{m-1} b(m-1, i) \sum_{r=(k+1)(m-1)-i+1}^{(k+1) m-i-1}(r-(k+1)(m-1)+i) \frac{(\lambda t)^{r}}{r !} \\
+e^{-\lambda t} \sum_{i=0}^{m-1} b(m-1, i) \sum_{r=(k+1) m-i}^{\infty} \frac{(\lambda t)^{r}}{r !}
\end{gathered}
$$

Now we rewrite the double-summation expressions in (A5), interchanging the order of summation of indices $i$ and $r$.

Consider the first double summation in (A5). By interchanging the order of summation, then index $r$ goes from $r=k(m-1)+1$ to $(k+1) m-1$. For a fixed $r$ within this range then index $i$ goes from $\max \{0,(k+1)(m-1)+1-r$ to $m-1-\max \{r-k m, 0\}$.

Similarly by changing the order of summation, the second double-summation expression in (A5) can, using that $\sum_{i=0}^{m-1} b(m-1, i)=1$, be rewritten to

$$
\sum_{r=k m+1}^{(k+1) m-1}\left[\sum_{i=(k+1) m-r}^{m-1} b(m-1, i)\right] \frac{(\lambda t)^{r}}{r !}+\sum_{r=(k+1) m}^{\infty} \frac{(\lambda t)^{r}}{r !}
$$

Therefore (A5) can be rewritten to

$$
\begin{array}{r}
\tilde{\Omega}_{m}(t)=\frac{e^{-\lambda t}}{k+1-p} \sum_{r=k(m-1)+1}^{(k+1) m-1}\left[\sum_{i=\max \{(k+1)(m-1)-r+1,0\}}^{m-1-\max \{r-k m, 0\}} b(m-1, i)(r-(k+1)(m-1)+i)\right] \frac{(\lambda t)^{r}}{r !} \\
+e^{-\lambda t} \sum_{r=k m+1}^{(k+1) m-1}\left[\sum_{i=(k+1) m-r}^{m-1} b(m-1, i)\right] \frac{(\lambda t)^{r}}{r !}+e^{-\lambda t} \sum_{r=(k+1) m}^{\infty} \frac{(\lambda t)^{r}}{r !}
\end{array}
$$

Now introduce the coefficients $\tilde{\Gamma}_{m}(r)$, defined in $(5 \mathrm{a}-\mathrm{b})$. Then (A7) can be rewritten to (4b).

Now we consider the case $m=1$.

$$
\tilde{\Omega}_{1}(t)=\int_{0}^{t} \tilde{\omega}(x) d x
$$




$$
=\frac{\lambda}{k+1-p}\left[\sum_{j=0}^{k-1} \int_{0}^{t} e^{-\lambda x} \frac{(\lambda x)^{j}}{j !} d x+(1-p) \int_{0}^{t} e^{-\lambda x} \frac{(\lambda x)^{k}}{k !} d x\right]
$$

Before we proceed we show

$$
\int_{a}^{u} e^{-\lambda x} \frac{(\lambda x)^{j}}{j !} d x=\frac{1}{\lambda}\left[e^{-\lambda a} \sum_{i=0}^{j} \frac{(\lambda a)^{i}}{i !}-e^{-\lambda u} \sum_{i=0}^{j} \frac{(\lambda u)^{i}}{i !}\right]
$$

for all non-negative reals $a$ and $u$ (with $a<u$ ), and a non-negative integer $j$. Note, that by proving (A9) we verify (11). We consider the following two cases.

When $j=0$

$$
\int_{a}^{u} e^{-\lambda x} d x=\frac{1}{\lambda}\left[e^{-\lambda a}-e^{-\lambda u}\right]
$$

When $\mathrm{j}>0$

$$
\begin{aligned}
& \int_{a}^{u} e^{-\lambda x} \frac{(\lambda x)^{j}}{j !} d x=\left[-\frac{1}{\lambda} e^{-\lambda x} \frac{(\lambda x)^{j}}{j !}\right]_{a}^{u}+\int_{a}^{u} e^{-\lambda x} \frac{(\lambda x)^{j-1}}{(j-1) !} d x \\
& =\frac{1}{\lambda}\left[e^{-\lambda a} \frac{(\lambda a)^{j}}{j !}-e^{-\lambda u} \frac{(\lambda u)^{j}}{j !}\right]+\int_{a}^{u} e^{-\lambda x} \frac{(\lambda x)^{j-1}}{(j-1) !} d x \\
& =\ldots=\frac{1}{\lambda}\left[e^{-\lambda a} \sum_{i=0}^{j} \frac{(\lambda a)^{i}}{i !}-e^{-\lambda u} \sum_{i=0}^{j} \frac{(\lambda u)^{i}}{i !}\right]
\end{aligned}
$$

Furthermore, when $a=0$ then the right hand side of (A9) is $\frac{1}{\lambda}\left[1-e^{-\lambda u} \sum_{i=0}^{j} \frac{(\lambda u)^{i}}{i !}\right]$.

Therefore (A8) can be rewritten to

$$
\begin{aligned}
& \tilde{\Omega}_{1}(t)=\frac{k}{k+1-p}\left[\sum_{j=0}^{k-1} \frac{1}{\lambda}\left[1-e^{-\lambda t} \sum_{i=0}^{j} \frac{(\lambda t)^{i}}{i !}\right]+\frac{1-p}{\lambda}\left[1-e^{-\lambda t} \sum_{i=0}^{j} \frac{(\lambda t)^{i}}{i !}\right]\right] \\
& =1-\frac{e^{-\lambda t}}{k+1-p} \sum_{j=0}^{k-1} \sum_{i=0}^{j} \frac{(\lambda t)^{i}}{i !}-\frac{1-p}{k+1-p} e^{-\lambda t} \sum_{i=0}^{k} \frac{(\lambda t)^{i}}{i !}
\end{aligned}
$$




$$
\begin{aligned}
& =1-\frac{e^{-\lambda t}}{k+1-p} \sum_{i=0}^{k-1} \frac{(\lambda t)^{i}}{i !} \sum_{j=i}^{k-1} 1-\frac{1-p}{k+1-p} e^{-\lambda t} \sum_{i=0}^{k} \frac{(\lambda t)^{i}}{i !} \\
& =1-\frac{e^{-\lambda t}}{k+1-p} \sum_{i=0}^{k-1}(k-i) \frac{(\lambda t)^{i}}{i !}-\frac{1-p}{k+1-p} e^{-\lambda t} \sum_{i=0}^{k} \frac{(\lambda t)^{i}}{i !} \\
& =1-e^{-\lambda t} \sum_{i=0}^{k-1} \frac{(\lambda t)^{i}}{i !}+\frac{e^{-\lambda t}}{k+1-p} \sum_{i=1}^{k-1} i \frac{(\lambda t)^{i}}{i !}-\frac{1-p}{k+1-p} e^{-\lambda t} \frac{(\lambda t)^{k}}{k !} \\
& =\frac{e^{-\lambda t}}{k+1-p} \sum_{i=1}^{k} i \frac{(\lambda t)^{i}}{i !}+e^{-\lambda t} \sum_{i=k+1}^{\infty} \frac{(\lambda t)^{i}}{i !}
\end{aligned}
$$

This fits to the general description in (4b) with

$$
\tilde{\Gamma}_{1}(i)=\frac{i}{k+1-p} \quad i=1, \ldots, k
$$

\section{Appendix B}

\section{Derivation of expressions (6) and (7a-b)}

The case $m=0$.

$\tilde{p}_{0}(t)=1-\tilde{\Omega}_{1}(t)=e^{-\lambda t} \sum_{r=0}^{k}\left(1-\frac{r}{k+1-p}\right) \frac{(\lambda t)^{r}}{r !}$

This can be written as (6) with $\tilde{\gamma}_{0}(r)$ specified as (7a).

The case $m \geq 1$.

$\tilde{p}_{m}(t)=\tilde{\Omega}_{m}(t)-\tilde{\Omega}_{m+1}(t)$

Because both $\tilde{\Omega}_{m}(t)$ and $\tilde{\Omega}_{m+1}(t)$ have common multipliers (which is 1 ) of $e^{-\lambda t} \frac{(\lambda t)^{r}}{r !}$ for summation index $r \geq(k+1)(m+1)$, we only need to compare the ranges of the summation indices of $r$ for the "complicating" terms in $\tilde{\Omega}_{m}(t)$ and $\tilde{\Omega}_{m+1}(t)$, respectively.

Here are the ranges of the summation indices $r=k(m-1)+1$ to $(k+1) m-1$ for $\tilde{\Omega}_{m}(t)$, and $r$ $=k m+1$ to $(k+1)(m+1)-1$ for $\tilde{\Omega}_{m+1}(t)$. 
The ranges overlap when $(k+1) m-1 \geq k m+1$, that is, when $m \geq 2$. Therefore the case $m$ $=2$ can be specified by (6) and (7c), while the case $m=1$ can be specified by (6) and (7b).

\section{Appendix C}

\section{Derivation of (9a-b)}

We make a self-contained derivation of the cost-expression, following the approach in [4]. Note that our derivation is slightly more general than [4] because we let the review interval be specified by a variable $R$ and not scaled to 1 as in [4].Let $t_{0}$ be the time point where review takes place, bringing the inventory position at level $x$. As in [4] we focus on the total costs incurred in the time-interval from $t_{0}+L$ to $t_{0}+L+R$.

On time point $t_{0}+L+\tau$, where $0 \leq \tau \leq R$, the average on-hand inventory is

$$
O H(\tau)=\left\{\begin{array}{cc}
\sum_{y=0}^{x-1}(x-y) P\left(\tilde{D}_{(L+\tau)}=y\right) & x>0 \\
0 & x \leq 0
\end{array}\right.
$$

while the expected backlog is

$B L(\tau)=\left\{\begin{array}{rr}\sum_{y=x}^{\infty}(y-x) P\left(\tilde{D}_{(L+\tau)}=y\right) & x>0 \\ -x+E\left[\tilde{D}_{(L+\tau)}\right] & x \leq 0\end{array}\right.$

$$
=\left\{\begin{array}{cc}
\frac{\lambda}{k+1-p}(L+\tau) E[X]-x+\sum_{y=0}^{x}(x-y) P\left(\tilde{D}_{(L+\tau)}=y\right) & x>0 \\
-x+\frac{\lambda}{k+1-p}(L+\tau) E[X] & x \leq 0
\end{array}\right.
$$

The expected new backlog during the time interval the time-interval from $t_{0}+L$ to $t_{0}+L$ $+R$ is 


$$
B L(R)-B L(0)=\left\{\begin{array}{cc}
\mathrm{R} E[X] \frac{\lambda}{k+1-p}+\sum_{y=0}^{x-1}(x-y)\left[P\left(\tilde{D}_{(L+R)}=y\right)-P\left(\tilde{D}_{(L)}=y\right)\right] & x>0 \\
R E[X] \frac{\lambda}{k+1-p} & x \leq 0
\end{array}\right.
$$

According to [4] the total costs incurred in the time-interval from $t_{0}+L$ to $t_{0}+L+R$ is

$$
G(x)=h \int_{0}^{R} O H(\tau) d \tau+v \int_{0}^{R} B L(\tau) d \tau+\pi(B L(R)-B L(0))
$$

It holds that

$$
\int_{0}^{R} O H(\tau) d \tau=\left\{\begin{array}{cc}
\sum_{y=0}^{x-1}(x-y) \int_{0}^{R} P\left(\tilde{D}_{(L+\tau)}=y\right) d \tau & x>0 \\
0 & x \leq 0
\end{array}\right.
$$

and

$$
\int_{0}^{R} B L(\tau) d \tau=\left\{\begin{array}{cc}
\left(\frac{\lambda}{k+1-p}\left(L+\frac{1}{2} R\right) E[X]-x\right) R+\sum_{y=0}^{x-1}(x-y) \int_{0}^{R} P\left(\tilde{D}_{(L+\tau)}=y\right) d \tau & x>0 \\
\left(\frac{\lambda}{k+1-p}\left(L+\frac{1}{2} R\right) E[X]-x\right) R & x \leq 0
\end{array}\right.
$$

Substituting (C3),C(5) and C(6) into (C4) gives (9a-b). 


\section{Working Papers from Logistics/SCM Research Group}

L-2006-09 Christian Larsen \& Gudrun P. Kiesmüller: Developing a closed-form cost expression for an $(R, s, n Q)$ policy where the demand process is compound generalized Erlang.

L-2006-08 Eduardo Uchoa, Ricardo Fukasawa, Jens Lysgaard, Artur Pessoa, Marcus Poggi de Aragão, Diogo Andrade: Robust Branch-Cut-and-Price for the Capacitated Minimum Spanning Tree Problem over a Large Extended Formulation.

L-2006-07 Geir Brønmo, Bjørn Nygreen \& Jens Lysgaard: Column generation approaches to ship scheduling with flexible cargo sizes.

L-2006-06 Adam N. Letchford, Jens Lysgaard \& Richard W. Eglese: A Branch-andCut Algorithm for the Capacitated Open Vehicle Routing Problem.

L-2006-05 Ole Mortensen \& Olga W. Lemoine: Business integration between manufacturing and transport-logistics firms.

L-2006-04 Christian H. Christiansen \& Jens Lysgaard: A column generation approach to the capacitated vehicle routing problem with stochastic demands.

L-2006-03 Christian Larsen: Computation of order and volume fill rates for a base stock inventory control system with heterogeneous demand to investigate which customer class gets the best service.

L-2006-02 Søren Glud Johansen \& Anders Thorstenson: Note: Optimal base-stock policy for the inventory system with periodic review, backorders and sequential lead times.

L-2006-01 Christian Larsen \& Anders Thorstenson: A comparison between the order and the volume fill rates for a base-stock inventory control system under a compound renewal demand process.

L-2005-02 Michael M. Sørensen: Polyhedral computations for the simple graph partitioning problem.

L-2005-01 Ole Mortensen: Transportkoncepter og IT-støtte: et undersøgelsesoplæg og nogle foreløbige resultater.

L-2004-05 Lars Relund Nielsen, Daniele Pretolani \& Kim Allan Andersen: K shortest paths in stochastic time-dependent networks.

L-2004-04 Lars Relund Nielsen, Daniele Pretolani \& Kim Allan Andersen: Finding the $K$ shortest hyperpaths using reoptimization.

L-2004-03 Søren Glud Johansen \& Anders Thorstenson: The $(r, q)$ policy for the lostsales inventory system when more than one order may be outstanding. 
L-2004-02 Erland Hejn Nielsen: Streams of events and performance of queuing systems: The basic anatomy of arrival/departure processes, when focus is set on autocorrelation.

L-2004-01 Jens Lysgaard: Reachability cuts for the vehicle routing problem with time windows. 
ISBN 87-7882-134-7

Department of Business Studies

Aarhus School of Business

Fuglesangs Allé 4

DK-8210 Aarhus V - Denmark

Tel. +4589486688

Fax +4586150188

www.asb.dk 